\title{
The Optimal Medical Therapy for Glycemic Control in COVID-19
}

\author{
Hidekatsu Yanai
}

\begin{abstract}
Diabetes is highly linked to the severity of coronavirus disease 2019 (COVID-19). My recent meta-analysis also suggested a higher prevalence of diabetes in severe COVID-19 as compared with non-severe COVID-19. Recent observational studies have shown that hyperglycemia was significantly associated with severity of COVID-19 in both diabetic and non-diabetic patients. To prevent worse outcome of COVID-19, more tight glucose control is required. I studied the association between hyperglycemia and worse outcome of COVID-19, the putative beneficial and harmful effects, and clinical outcomes of oral hypoglycemic drugs and insulin use in glycemic control among COVID-19 patients, by searching literatures. Although there were some negative studies, the meta-analysis reported that the treatment using metformin was associated with reduction in mortality due to COVID-19. One study showed that treatment with sitagliptin, one of dipeptidyl peptidase-4 (DPP4) inhibitors, during hospitalization was associated with reduction of mortality, with a clinical improvement as compared with patients on the standard care. There were no clinical studies showed effects of glucagon-like peptide-1 receptor agonists, pioglitazone and sulfonylurea on COVID-19 outcomes. Regarding sodium-glucose cotransporter 2 (SGLT2) inhibitors, a case of euglycemic diabetic ketoacidosis (DKA) associated with COVID-19 and a case of DKA that was difficult to distinguish from COVID-19 were reported. COVID-19 patients who need hospital care may deteriorate rapidly, an early and appropriate initiation of insulin therapy in hyperglycemic COVID-19 patients may be to be encouraged.
\end{abstract}

Keywords: Glucose control; COVID-19; Oral hypoglycemic drugs; Insulin therapy

\section{Introduction}

Coronavirus disease 2019 (COVID-19) induced by severe

Manuscript submitted December 18, 2020, accepted December 29, 2020

Published online February 3, 2021

Department of Diabetes, Endocrinology and Metabolism, National Center for Global Health and Medicine Kohnodai Hospital, 1-7-1 Kohnodai, Ichikawa, Chiba 272-8516, Japan. Email: dyanai@hospk.ncgm.go.jp

doi: https://doi.org/10.14740/jem718 acute respiratory syndrome coronavirus 2 (SARS-CoV-2) has reached a pandemic level. Diabetes is one of the most important comorbidities linked to the severity of COVID-19. My meta-analysis of 13 studies showed that the existence of diabetes was significantly associated with severity of COVID-19 [1]. A recent study involving COVID-19 patients admitted to the intensive care unit (ICU) $(\mathrm{n}=495)$ showed that hyperglycemia was significantly associated with prolonged ICU stay, mechanical ventilation use, and higher mortality [2]. Tighter blood glycemic control is warranted to COVID-19 patients.

Here, I will show the association between hyperglycemia and worse outcome of COVID-19, the putative beneficial and harmful effects, and clinical outcomes of oral hypoglycemic drugs and insulin use in glycemic control among COVID-19 patients, by searching literatures.

\section{Hyperglycemia and Worse Outcome of COV- ID-19}

The activation of the hypothalamic-pituitary-adrenal axis by severe infection induces over-secretion of insulin-counteracting hormones such as cortisol, growth hormone and glucagon, which increases plasma glucose. Furthermore, inflammatory cytokines induce insulin resistance [3]. A multi-center retrospective study showed that mortality and mechanical ventilation use were significantly more frequent in the hyperglycemic group (average blood glucose $(\mathrm{BG})>$ and $=140 \mathrm{mg} / \mathrm{dL}$ ) than the normoglycemic group (average BG $<140 \mathrm{mg} / \mathrm{dL})(31.4 \%$ vs. $16.6 \% ; 50.0 \%$ vs. $37.2 \%$, respectively) [2]. Hyperglycemia (hazard ratio (HR), 1.79; 95\% confidence interval (CI), 1.14 - 2.82) predicted the in-hospital mortality. Steroids use (odds ratio (OR), 1.521; 95\% CI, $1.054-2.194)$ and triglycerides $>150 \mathrm{mg} / \mathrm{dL}$ (OR, 1.62; 95\% CI, 1.109 - 2.379) on admission were associated with hyperglycemia [2]. Yang et al studied the association between fasting plasma glucose (FPG) and 28-day mortality in COVID-19 patients [4]. Higher levels of neutrophil counts and D-dimer, and lower levels of lymphocyte counts and albumin, and higher incidence of fatal complications were observed in patients with diabetes. FPG with $7.0-11.0$ (HR, 1.90; 95\% CI, $1.11-3.25)$ and $\geq 11.1 \mathrm{mmol} / \mathrm{L}$ (HR, 2.09; 95\% CI, 1.21 - 3.64) were independent predictors of 28-day mortality [4]. A pooled analysis on the association between hyperglycemia and outcomes of COVID-19 showed that hyperglycemia was significantly associated with higher incidence of critical illness (OR, 1.837; 95\% CI, 1.368 - 2.465) 
Table 1. Putative Beneficial and Harmful Effects, and Clinical Outcomes of Metformin Use in Glycemic Control Among COVID-19 Patients

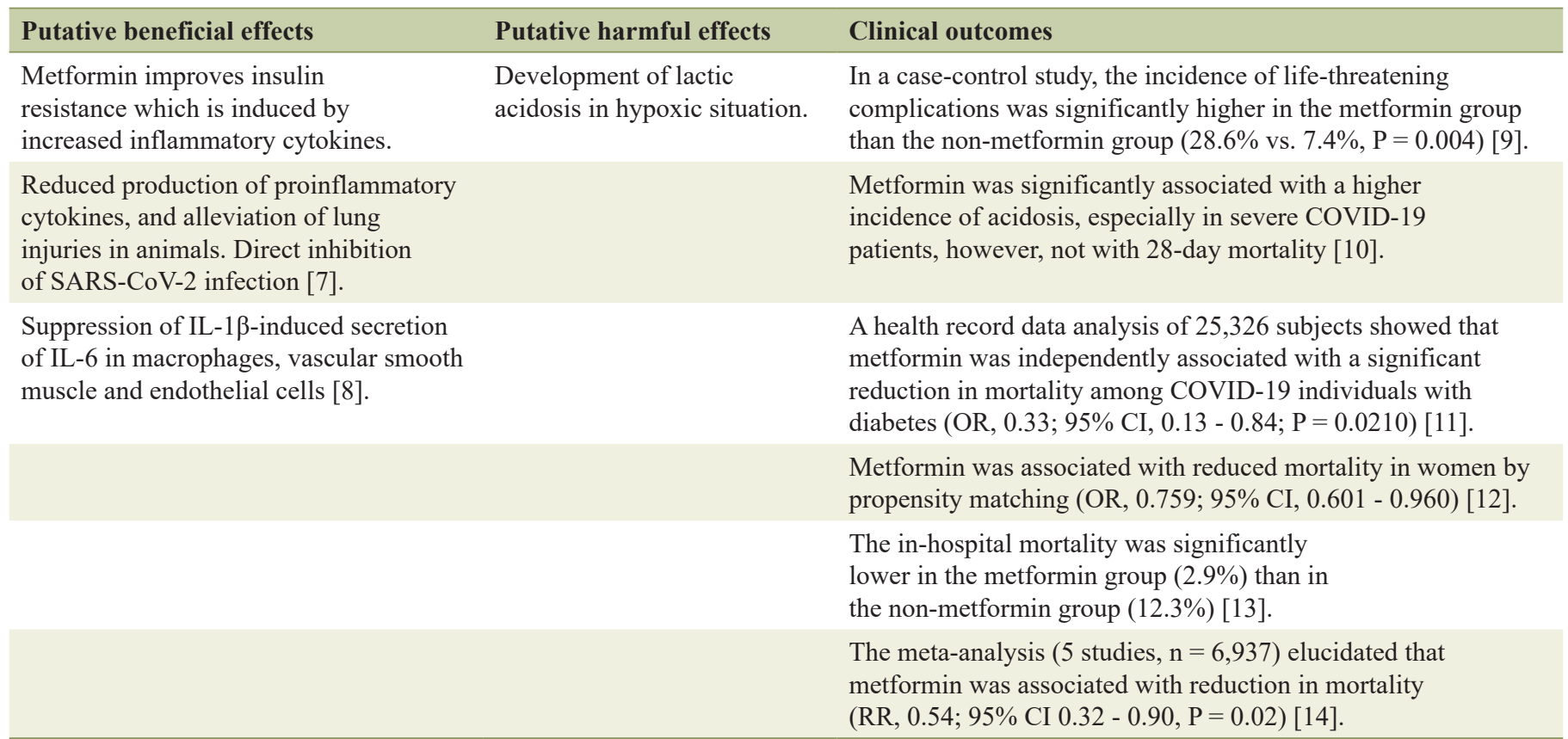

COVID-19: coronavirus disease 2019; SARS-CoV-2: severe acute respiratory syndrome coronavirus 2; RR: relative risk; IL: interleukin; Cl: confidence interval.

and mortality (OR, 2.822; 95\% CI, 1.587 - 5.019) than normal blood glucose levels, in non-diabetic patients [5]. In a retrospective study in Spain $(\mathrm{n}=11,312)$, the in-hospital mortality rates were $15.7 \%$ in patients with $\mathrm{BG}<140 \mathrm{mg} / \mathrm{dL}, 33.7 \%$ in patients with BG $140-180 \mathrm{mg} / \mathrm{dL}$ and $41.1 \%$ in patients with $\mathrm{BG}>180 \mathrm{mg} / \mathrm{dL}[6]$. Hyperglycemia was significantly associated with mechanical ventilation use, ICU stay and mortality [6].

Hyperglycemia was significantly associated with severity of COVID-19 in both diabetic and non-diabetic patients. To prevent worse outcome of COVID-19, more tight glucose control is required.

\section{Putative Beneficial and Harmful Effects, and Clinical Outcomes of Oral Hypoglycemic Drugs Use in Glycemic Control Among COVID-19 Pa- tients}

\section{Metformin}

Metformin, a drug for the treatment of type 2 diabetes, reduces the proinflammatory cytokines production (Table 1 [7-14]). Alleviation of lung injuries by metformin was observed in animals and a direct inhibition of SARS-CoV-2 infection by metformin has been supposed [7].

Metformin was reported to inhibit interleukin-6 (IL-6) release in mucosal cells, macrophages, vascular smooth muscle and endothelial cells [8]. For glycemic control, metformin improves insulin resistance which is induced by increased inflammatory cytokines.

As a putative harmful effect, development of lactic acidosis in hypoxic situation such as acute respiratory distress syndrome (ARDS) and severe pneumonia should be considered.

In a case-control study conducted by Gao et al, the incidence of life-threatening complications was significantly higher in the metformin group than the non-metformin group (28.6\% vs. $7.4 \%, \mathrm{P}=0.004)$ [9]. In a retrospective study $(\mathrm{n}=$ $1,213)$, the treatment using metformin was significantly associated with the development of acidosis, especially in individuals with severe COVID-19, however, not with 28-day mortality [10]. A health record data analysis $(n=25,326)$ showed that metformin use was independently and significantly associated with decrease of mortality in diabetic patients with COVID-19 (OR, 0.33; 95\% CI, $0.13-0.84$; P = 0.021) [11]. Further, metformin use was associated with reduced mortality in women by propensity matching (OR, 0.759; 95\% CI, 0.601 - 0.960) [12]. Another observational study demonstrated that the in-hospital mortality was significantly lower in the metformin group $(2.9 \%)$ than in the non-metformin group (12.3\%) [13]. The meta-analysis (five studies; $\mathrm{n}=6,937$ ) showed that metformin was associated with decrease of mortality (relative risk (RR), $0.54 ; 95 \%$ CI, $0.32-0.90, \mathrm{P}=0.02$ ) [14].

One case-control study showed a significant association between metformin and critical complications. Another observational study reported an association of metformin with the development of acidosis in severe COVID-19, but not with mortality. Other three studies showed that metformin use was associated with a significant reduction in mortality in COV- 
Table 2. Putative Beneficial and Harmful Effects, and Clinical Outcomes of Incretin-Related Drugs Use in Glycemic Control Among COVID-19 Patients

\begin{tabular}{lll}
\hline Putative beneficial effects & Putative harmful effects & Clinical outcomes \\
\hline DPP4 inhibitors are unlikely to develop hypoglycemia. & No applicable data & $\begin{array}{l}\text { Sitagliptin use during hospitalization was } \\
\text { associated with reduced mortality }(\mathrm{HR}, \\
0.44 ; 95 \% \mathrm{CI}, 0.29-0.66 ; \mathrm{P}=0.0001), \\
\text { with clinical improvement }(60 \% \text { vs. } \\
38 \% ; \mathrm{P}=0.0001) \text { and with more hospital } \\
\text { discharges }(120 \text { vs. } 89 ; \mathrm{P}=0.0008) \text { as } \\
\text { compared with the standard care }[19] .\end{array}$ \\
\hline
\end{tabular}

DPP4 inhibitors increase secretion of insulin, anabolic hormone, which may be beneficial for the treatment of severe infection.

The identified DPP4 networks are highly enriched in viral processes required for viral entry and infection, therefore, DPP4 was proposed as an important putative target for the treatment of COVID-19 [15].

A meta-analysis reported a significant reduced CRP levels by DPP4 inhibitors as compared with placebo [2].

Sitagliptin was reported to improve inflammatory status by affecting on NF-kappa-B signaling [16].

The S1 domain of SARS-CoV-2 spike glycoprotein potentially interacts with DPP4 [17]. DPP4 inhibitors can be the therapeutic candidate for COVID-19 by blocking the entry of SARS-CoV-2 into human body [18].

In type 2 diabetic patients, liraglutide (GLP-1 RA) significantly reduced serum CRP levels [2].

GLP-1 RA: glucagon-like peptide-1 receptor agonist; HR: hazard ratio; COVID-19: coronavirus disease 2019; DPP4: dipeptidyl peptidase-4; CRP: C-reactive protein.

ID-19, and the meta-analysis also showed that metformin was associated with decrease of mortality.

\section{Incretin-related drugs}

Dipeptidyl peptidase-4 (DPP4) inhibitors increase secretion of insulin, anabolic hormone, which may be beneficial for the treatment of severe infection (Table 2 [2, 15-19]). DPP4 inhibitors are unlikely to develop hypoglycemia.

The identified DPP4 networks are highly enriched in viral processes required for viral entry and infection, and DPP4 was proposed as an important putative target for the treatment of COVID-19 [15]. DPP4 inhibitors may play an important role in immune functions and inflammation. A recent metaanalysis reported a significant decrease of C-reactive protein (CRP) levels by DPP4 inhibitors as compared with placebo [2]. DPP4 has been supposed to be associated with regulation of antibody production, secretion of cytokines. Sitagliptin has been reported to reduce inflammation levels mostly by affecting on NF-kappa-B signaling [16].

The S1 domain of SARS-CoV-2 spike glycoprotein potentially interacts with DPP4 [17]. DPP4 is widely expressed on blood vessels, myocardium, and myeloid cells, and is upregulated by proinflammatory states [20]. DPP4 inhibitors can be the therapeutic candidate for COVID-19 by blocking the entry of SARS-CoV-2 into human body [18].

Glucagon-like peptide-1 receptor agonists (GLP-1 RAs) have anti-inflammatory properties. In type 2 diabetic patients receiving liraglutide, CRP was significantly decreased [2].

In a multicenter study, sitagliptin was added to the standard care during hospitalization in COVID-19 patients with type 2 diabetes [19]. Of 338 patients, 169 were on sitagliptin, while 169 were on the standard care. Sitagliptin use was significantly associated with reduction of mortality (HR, 0.44; 95\% CI, 0.29 - 0.66), with an improvement of COVID-19 (60\% vs. 38\%; P= $0.0001)$ as compared with patients on the standard care.

\section{Pioglitazone}

IL-6 expression was reduced by pioglitazone in monocytes, white adipose tissue and cardiomyocytes (Table 3 [2, 21-24]). Pioglitazone significantly reduced serum CRP levels. A previous meta-analysis reported that pioglitazone significantly reduced high-sensitivity CRP levels in type 2 diabetic patients [2]. Progressive atherosclerosis may be associated with the development of COVID-19. Oxidized LDL (ox-LDL) plays a crucial role in atherogenesis. Uptake of ox-LDL by macrophages activates the reprogramming of innate immunity, which is termed "trained immunity." Ox-LDL-trained macrophages may induce over-secretion of cytokines triggered by 
Table 3. Putative Beneficial and Harmful Effects, and Clinical Outcomes of Pioglitazone and Sulfonylurea in Glycemic Control Among COVID-19 Patients

\begin{tabular}{|c|c|c|}
\hline Putative beneficial effects & Putative harmful effects & Clinical outcomes \\
\hline \multicolumn{3}{|l|}{ Pioglitazone } \\
\hline $\begin{array}{l}\text { IL-6 expression was suppressed by pioglitazone in } \\
\text { monocytes, white adipose tissue and cardiomyocytes. } \\
\text { Pioglitazone significantly reduced serum CRP levels. A } \\
\text { meta-analysis demonstrated that pioglitazone significantly } \\
\text { lowered serum hs-CRP levels in type } 2 \text { diabetic patients [2]. }\end{array}$ & $\begin{array}{l}\text { As my opinion, pioglitazone may possibly } \\
\text { exacerbate pulmonary congestion or acute } \\
\text { respiratory distress syndrome (ARDS) } \\
\text { which need critical management of body } \\
\text { fluid by increasing fluid retention. }\end{array}$ & No applicable data \\
\hline \multicolumn{3}{|l|}{$\begin{array}{l}\text { Progressive atherosclerosis is associated with the } \\
\text { development of severe COVID-19. Oxidized LDL (Ox- } \\
\text { LDL) plays an important role for atherogenesis. Uptake } \\
\text { of ox-LDL by macrophages activates the reprogramming } \\
\text { of innate immunity, "trained immunity." Ox-LDL- } \\
\text { trained macrophages infected with SARS-CoV-2 increase } \\
\text { cytokine release, which leads to lung injury. Inhibition of } \\
\text { macrophage training by pioglitazone may suppress the } \\
\text { cytokine storm induced by SARS-CoV-2 infection [21]. }\end{array}$} \\
\hline $\begin{array}{l}\text { Sulfonylurea increases secretion of insulin, } \\
\text { anabolic hormone, which may be beneficial } \\
\text { for the treatment of severe infection. }\end{array}$ & $\begin{array}{l}\text { Sulfonylurea is likely to } \\
\text { develop hypoglycemia. }\end{array}$ & $\begin{array}{l}\text { A combination therapy } \\
\text { of metformin and } \\
\text { sulfonylureas was most } \\
\text { commonly associated } \\
\text { with the development } \\
\text { of hypoglycemia } \\
(65.75 \%) \text { [24]. }\end{array}$ \\
\hline \multicolumn{3}{|l|}{$\begin{array}{l}\text { Channel activity was found for the envelope } \\
\text { protein of coronaviruses, and the ion channel } \\
\text { was inhibited by gliclazide [22]. }\end{array}$} \\
\hline $\begin{array}{l}\text { Glibenclamide presented 3D similarity to known } \\
\text { ligands, reasonable predicted binding modes and } \\
\text { micromolar predicted binding affinity values } \\
\text { for the SARS-CoV-2 main protease [23]. }\end{array}$ & & \\
\hline
\end{tabular}

COVID-19: coronavirus disease 2019; SARS-CoV-2: severe acute respiratory syndrome coronavirus 2; IL: interleukin; hs-CRP: high-sensitivity Creactive protein.

SARS-CoV-2 infection in the lung. Therefore, the pioglitazone-mediated inhibition of macrophage training could reduce the cytokines-induced lung injuries [21].

As my opinion, pioglitazone may possibly exacerbate pulmonary congestion or ARDS which need critical management of body fluid by increasing fluid retention.

\section{Sulfonylurea}

Sulfonylurea increases secretion of insulin, anabolic hormone, which may be beneficial for the treatment of severe infection (Table 3 [2, 21-24]). However, sulfonylurea is likely to develop hypoglycemia.

Channel activity was found for the envelope protein of coronaviruses, and the ion channel was inhibited by gliclazide [22]. Essential for viral replication, the main protease has been explored as a promising target for the drug discovery process. The virtual screening pipeline relies on the known 3D properties of noncovalent ligands and features of crystalized complexes, applying consensus analyses in each step. Glibenclamide presented 3D similarity to known ligands, reasonable predicted binding modes and micromolar predicted binding affinity values. Glibenclamide was identified as promising inhibitor of the main viral protease [23].

Shah et al analyzed the data retrospectively from 146 patients of type 2 diabetes reporting to the emergency department during lockdown period with symptoms suggestive of hypoglycemia [24]. A combination of metformin and sulfonylureas was most commonly associated with the development of hypoglycemia $(65.75 \%)$ in patients with type 2 diabetes during the COVID-19 lockdown [24].

\section{Sodium-glucose cotransporter 2 (SGLT2) inhibitors}

Owing to their pharmacological characteristics, SGLT2 inhibitors might cause adverse effects in patients with COVID-19 
Table 4. Putative Beneficial and Harmful Effects, and Clinical Outcomes of SGLT2 Inhibitors in Glycemic Control Among COVID-19 Patients

\begin{tabular}{ll}
\hline Putative beneficial effects & Putative harmful effects \\
\hline No applicable data & Owing to their pharmacological characteristics, \\
& SGLT2 inhibitors might cause adverse \\
effects in patients with COVID-19 and \\
so cannot be recommended [25].
\end{tabular}

People with diabetes should be encouraged to Clinical outcomes

A 52-year-old male with type 2 diabetes on empagliflozin and no history of diabetic ketoacidosis (DKA) presented with symptoms of COVID-19 as well as laboratory findings consistent with euglycemic DKA. SGLT2 inhibitors should be held as early as possible in COVID-19 patients due to the risk of euglycemic DKA [28]. continue medication prescribed for hypertension, The patient of euglycemic DKA due to empagliflozin use diabetes or dyslipidemia. Furthermore, patients was initially suspected to be a case of COVID-19 [29]. with diabetes and COVID-19 infection should follow their usual antidiabetic treatment with the exception of SGLT2 inhibitors [26].

Avoidance of diabetic ketoacidosis associated with SGLT2 inhibitors is of particular medical importance during the COVID-19 pandemic [27].

COVID-19: coronavirus disease 2019; SGLT2: sodium-glucose cotransporter 2.

and so cannot be recommended [25] (Table 4 [25-29]). People with diabetes should be encouraged to continue medication prescribed for hypertension, diabetes or dyslipidemia. Furthermore, patients with diabetes and COVID-19 should follow their usual antidiabetic treatment with the exception of SGLT2 inhibitors [26]. Avoidance of diabetic ketoacidosis associated with SGLT2 inhibitors is of particular medical importance during the COVID-19 pandemic [27].

A 52-year-old male with type 2 diabetes on empagliflozin and no history of diabetic ketoacidosis (DKA) presented with symptoms of COVID-19 as well as laboratory findings consistent with euglycemic DKA. SGLT2 inhibitors should be held as early as possible in COVID-19 patients due to the risk of euglycemic DKA [28]. The patient of euglycemic DKA due to empagliflozin use was initially suspected to be a case of COVID-19 [29]. During the pandemic of COVID-19, euglycemic DKA due to SGLT2 inhibitors may disrupt emergency medical care.

\section{Insulin Therapy in Glycemic Control Among COVID-19 Patients}

Hyperglycemia was significantly associated with severity of COVID-19 in both diabetic and non-diabetic patients. To prevent worse outcome of COVID-19, more tight glucose control is required. To obtain prompt and tight glycemic control, insulin therapy may be useful.

In the retrospective study investigating 689 patients with COVID-19 and type 2 diabetes, insulin therapy was associated with a significant increase in mortality (adjusted HR, 5.38; 95\% CI, 2.75 - 10.54) [30]. Insulin therapy was associated with enhanced systemic inflammation and aggravated injuries of vital organs. We should not conclude that insulin treatment is harmful for glycemic control in COVID-19 by this result. The need for insulin may reflect the severity of COVID-19 and the duration of diabetes.

Agarwal et al studied pre-existing risk factors for mortality in hospitalized COVID-19 patients with diabetes [31]. Insulin therapy that reflects the duration of diabetes was predictive of mortality [31].

In the analysis of 562 ICU patients, the time in range (TIR) $(70-150 \mathrm{mg} / \mathrm{dL})$ and the daily insulin dose in critical COVID-19 patients and non-COVID-19 patients were investigated [32]. The TIR in COVID-19 patients (44.4\%) was significantly lower than non-COVID-19 patients $(68.5 \%)$. COVID-19 patients needed more daily insulin dose ( 8.37 units) than non-COVID-19 patients (6.17 units). A lower TIR was associated with ventilator use, and a higher TIR was associated with lower mortality, suggesting a significant association between poor glycemic control and poor COVID-19 outcomes among ICU patients.

Wu et al analyzed characteristics, insulin therapy and inflammatory markers of diabetic patients admitted to ICU with COVID-19 [33]. During highly inflammatory state, extremely high insulin doses (2.2 units/kg/day) were required for glycemic control. With reduced inflammation, daily insulin requirement decreased and glycemic control improved.

COVID-19 patients who need hospitalization seemed to deteriorate rapidly; therefore, an early initiation of an appropriate insulin therapy in hyperglycemic patients with COVID-19 is to be encouraged [34]. Considering various studies, the target glucose range in COVID-19 patients may be $140-180 \mathrm{mg} /$ $\mathrm{dL}$ [35]. Beyond the control of hyperglycemia, an early initiation of insulin therapy is considered to have beneficial effects for immunomodulation, regulate the renin-angiotensin system (RAS) which can induce acute lung injury [36].

During highly inflammatory status due to COVID-19, the achievement of an appropriate glycemic control is difficult and needs high doses of insulin. To reduce deaths and severe cases due to COVID-19, it is important to obtain good glycemic control even with a large amount of insulin from an earlier stage. 


\section{Conclusions}

Although there were two negative studies, the meta-analysis showed that metformin use was associated with reduction in mortality of COVID-19 patients. One study showed that sitagliptin was associated with reduction of mortality and clinical improvement compared with the standard care. There were no clinical studies showed effects of GLP-1 RAs, pioglitazone and sulfonylurea on COVID-19 clinical outcomes. Regarding SGLT2 inhibitors, a case of euglycemic DKA associated with COVID-19 and a case of DKA that was difficult to distinguish from COVID-19 were reported. COVID-19 patients who need hospitalization seemed to deteriorate rapidly, therefore, an early initiation of an appropriate insulin therapy in hyperglycemic patients with COVID-19 is to be encouraged.

\section{Acknowledgments}

Not applicable.

\section{Financial Disclosure}

Author has no financial disclosure to report.

\section{Conflict of Interest}

The author declares that he has no conflict of interest concerning this article.

\section{Informed Consent}

Not applicable.

\section{Author Contributions}

H.Y. designed the research, and collected and analyzed data. H.Y. wrote and approved the final paper.

\section{Data Availability}

The data supporting the findings of this study are available from the corresponding author upon reasonable request.

\section{References}

1. Yanai H. A significance of high prevalence of diabetes and hypertension in severe COVID-19 patients. J Clin Med Res. 2020;12(6):389-392.

2. Saand AR, Flores M, Kewan T, Alqaisi S, Alwakeel M, Griffiths L, Wang X, et al. Does inpatient hyperglycemia predict a worse outcome in COVID-19 intensive care unit patients? J Diabetes. 2020.

3. Kolyva AS, Zolota V, Mpatsoulis D, Skroubis G, Solomou EE, Habeos IG, Assimakopoulos SF, et al. The role of obesity in the immune response during sepsis. Nutr Diabetes. 2014;4:e137.

4. Yang P, Wang N, Wang J, Luo A, Gao F, Tu Y. Admission fasting plasma glucose is an independent risk factor for 28-day mortality in patients with COVID-19. J Med Virol. 2020.

5. Sachdeva S, Desai R, Gupta U, Prakash A, Jain A, Aggarwal A. Admission hyperglycemia in non-diabetics predicts mortality and disease severity in COVID-19: a pooled analysis and meta-summary of literature. SN Compr Clin Med. 2020:1-6.

6. Carrasco-Sanchez FJ, Lopez-Carmona MD, MartinezMarcos FJ, Perez-Belmonte LM, Hidalgo-Jimenez A, Buonaiuto V, Suarez Fernandez C, et al. Admission hyperglycaemia as a predictor of mortality in patients hospitalized with COVID-19 regardless of diabetes status: data from the Spanish SEMI-COVID-19 Registry. Ann Med. 2021;53(1):103-116.

7. Do JY, Kim SW, Park JW, Cho KH, Kang SH. Is there an association between metformin use and clinical outcomes in diabetes patients with COVID-19? Diabetes Metab. 2020.

8. Katsiki N, Ferrannini E. Anti-inflammatory properties of antidiabetic drugs: A "promised land" in the COVID-19 era? J Diabetes Complications. 2020;34(12):107723.

9. Gao Y, Liu T, Zhong W, Liu R, Zhou H, Huang W, Zhang $\mathrm{W}$. Risk of metformin in patients with type 2 diabetes with COVID-19: a preliminary retrospective report. Clin Transl Sci. 2020;13(6):1055-1059.

10. Cheng X, Liu YM, Li H, Zhang X, Lei F, Qin JJ, Chen $Z$, et al. Metformin Is Associated with Higher Incidence of Acidosis, but Not Mortality, in Individuals with COVID-19 and Pre-existing Type 2 Diabetes. Cell Metab. 2020;32(4):537-547 e533.

11. Crouse A, Grimes T, Li P, Might M, Ovalle F, Shalev A. Metformin use is associated with reduced mortality in a diverse population with COVID-19 and diabetes. medRxiv. 2020.

12. Bramante $\mathrm{C}$, Ingraham $\mathrm{N}$, Murray $\mathrm{T}$, Marmor $\mathrm{S}$, Hoversten S, Gronski J, McNeil C, et al. Observational Study of Metformin and Risk of Mortality in Patients Hospitalized with Covid-19. medRxiv. 2020.

13. Luo P, Qiu L, Liu Y, Liu XL, Zheng JL, Xue HY, Liu WH, et al. Metformin Treatment Was Associated with Decreased Mortality in COVID-19 Patients with Diabetes in a Retrospective Analysis. Am J Trop Med Hyg. 2020;103(1):69-72.

14. Hariyanto TI, Kurniawan A. Metformin use is associated with reduced mortality rate from coronavirus disease 2019 (COVID-19) infection. Obes Med. 2020;19:100290.

15. Bardaweel SK, Hajjo R, Sabbah DA. Sitagliptin: a potential drug for the treatment of COVID-19? Acta Pharm. 2021;71(2):175-184.

16. Mozafari N, Azadi S, Mehdi-Alamdarlou S, Ashrafi H, Azadi A. Inflammation: A bridge between diabetes and 
COVID-19, and possible management with sitagliptin. Med Hypotheses. 2020;143:110111.

17. Vankadari N, Wilce JA. Emerging WuHan (COVID-19) coronavirus: glycan shield and structure prediction of spike glycoprotein and its interaction with human CD26. Emerg Microbes Infect. 2020;9(1):601-604.

18. Yanai H. Can dipeptidyl peptidase 4 inhibitor be the therapeutic candidate for the COVID-19? J Endocrinol Metab. 2020;10(2):35.

19. Solerte SB, D'Addio F, Trevisan R, Lovati E, Rossi A, Pastore I, Dell'Acqua M, et al. Sitagliptin treatment at the time of hospitalization was associated with reduced mortality in patients with type 2 diabetes and COVID-19: a multicenter, case-control, retrospective, observational study. Diabetes Care. 2020;43(12):2999-3006.

20. Zhong J, Maiseyeu A, Davis SN, Rajagopalan S. DPP4 in cardiometabolic disease: recent insights from the laboratory and clinical trials of DPP4 inhibition. Circ Res. 2015;116(8):1491-1504.

21. Erol A. Role of oxidized LDL-induced "trained macrophages" in the pathogenesis of COVID-19 and benefits of pioglitazone: A hypothesis. Diabetes Metab Syndr. 2020;14(4):713-714.

22. Singh Tomar PP, Arkin IT. SARS-CoV-2 E protein is a potential ion channel that can be inhibited by Gliclazide and Memantine. Biochem Biophys Res Commun. 2020;530(1):10-14.

23. Ferraz WR, Gomes RA, AL SN, Goulart Trossini GH. Ligand and structure-based virtual screening applied to the SARS-CoV-2 main protease: an in silico repurposing study. Future Med Chem. 2020;12(20):1815-1828.

24. Shah K, Tiwaskar M, Chawla P, Kale M, Deshmane R, Sowani A. Hypoglycemia at the time of Covid-19 pandemic. Diabetes Metab Syndr. 2020;14(5):1143-1146.

25. Lim S, Bae JH, Kwon HS, Nauck MA. COVID-19 and diabetes mellitus: from pathophysiology to clinical management. Nat Rev Endocrinol. 2021;17(1):11-30.

26. Doupis J, Avramidis K. Managing diabetes during the COVID-19 pandemic. Eur Endocrinol. 2020;16(2):85-
87.

27. Milder TY, Stocker SL, Day RO, Greenfield JR. Potential safety issues with use of sodium-glucose cotransporter 2 inhibitors, particularly in people with type 2 diabetes and chronic kidney disease. Drug Saf. 2020;43(12):12111221.

28. Fang J, Genco M, Caskey RN. COVID-19 Precipitating Euglycaemic diabetic ketoacidosis with SGLT2 inhibitor use. Eur J Case Rep Intern Med. 2020;7(11):001943.

29. Ozer O, Yorulmaz G. Euglycemic diabetic ketoacidosis associated with empagliflozin use in the course of the SARS-Cov-2 pandemic. J Coll Physicians Surg Pak. 2020;30(10):110-111.

30. Yu B, Li C, Sun Y, Wang DW. Insulin Treatment is associated with increased mortality in patients with COVID-19 and type 2 diabetes. Cell Metab. 2021;33(1):65-77 e62.

31. Agarwal S, Schechter C, Southern W, Crandall JP, Tomer Y. Preadmission diabetes-specific risk factors for mortality in hospitalized patients with diabetes and coronavirus disease 2019. Diabetes Care. 2020;43(10):2339-2344.

32. Kapoor R, Timsina LR, Gupta N, Kaur H, Vidger AJ, Pollander AM, Jacobi J, et al. Maintaining blood glucose levels in range (70-150 mg/dL) is difficult in COVID-19 compared to non-COVID-19 ICU patients-a retrospective analysis. J Clin Med. 2020;9(11):3635.

33. Wu L, Girgis CM, Cheung NW. COVID-19 and diabetes: Insulin requirements parallel illness severity in critically unwell patients. Clin Endocrinol (Oxf). 2020;93(4):390393.

34. Nakhleh A, Shehadeh N. Glycemic control of type 2 diabetic patients with coronavirus disease during hospitalization: a proposal for early insulin therapy. Am J Physiol Endocrinol Metab. 2020;318(6):E835-E837.

35. American Diabetes Association. 15. Diabetes Care in the Hospital: Standards of Medical Care in Diabetes-2020. Diabetes Care. 2020;43(Suppl 1):S193-S202.

36. Zhang H, Baker A. Recombinant human ACE2: acing out angiotensin II in ARDS therapy. Crit Care. 2017;21(1):305 